\title{
An experimental study to check immediate effect of stacked breathing exercise on peak expiratory flow rate in cardiac surgery patient-pilot study
}

\begin{abstract}
Aims and Objectives: To evaluate the immediate effect of Stacked Breathing Exercise (SBE) on Peak Expiratory Flow (PEFR) in cardiac surgery patient.

Methodology: After obtaining an ethical committee clearance, a pilot study is carried out on 11 subjects with age 40$70 y$ rs planned for cardiac surgery through the mediastinal approach. After obtaining written consent and screening of patient for the inclusion, Patients were treated with a Stacked Breathing Exercise (SBE) on Post-Operative Day (POD) 1, 2 and 3 twice daily. Pre-exercise and post-exercise values for PEFR was collected in six treatment session as IA, IB, IIA, IIB, IIIA, IIIB ( $A=$ Morning session, B=Afternoon session and I, II, III was POD-1, 2 and 3 respectively).

Result: Total of 11 patients followed by cardiac surgery are analyzed for outcome PEFR. Data was analyzed in term of the mean difference. There is a statistically significant change seen in PEFR value comparing IA vs. IIIA, IB vs. IIIB and IA vs. IIIB (mean difference $-86.364,-73.636$ and -106.36 ). There is net $29.77 \%$ improvement in PEFR value seen at the end of the sixth (IIB) treatment session over pre-exercise value at IA.

Discussion: Statistically significant improvement seen in PEFR value when comparing pre-exercise value to postexercise with every session immediately after the intervention. Our result is best supported with research article carried out by, Luciana Chiavegato. He said, Breath Stacking as an intervention in non-cardiac thoracic surgery patients able to show significant improvement in the Peak Expiratory Flow (PEF). We conducted this study to have to check on, whether it is also effective in cardiac surgery patients? In our study, we found $27 \%$ gain over before exercise values of PEFR (in I/ $\mathrm{min}$ ) after stacked breathing exercise in cardiac surgery patients.
\end{abstract}

Conclusion: It is concluded that stacked breathing exercise is significantly effective in improving PEFR. Hence can be an effective tool to help in improving lung function.

Keywords: stacked breathing exercise (SBE), cardiac surgery with mediastinal approach, cardiac surgery patient, Peak expiratory flow rate (PEFR)

\section{Introduction}

India has recently taken over the position of being the most populous country in the world. Nowadays Ischemic Heart Disease (IHD) remains the number one cause of mortality and it is alarming to note that three of the six top causes of death are from respiratory complications $[1,2]$. Cardiac surgery through a sternotomy has deleterious effects on the function of the respiratory muscle action and the chest wall $[3,4]$. Patients develop restrictive respiratory abnormalities with reduced lung volumes and impaired gas exchange in the early postoperative period [5,6]. Atelectasis, bronchospasm, dyspnea, cough, pneumonia, respiratory failure, and exacerbation of lung disease have all been reported as complications $[3,4]$.

Physiotherapy has been widely used as a means of improving postoperative pulmonary function and reducing complications $[7,8]$. Chest physiotherapy techniques most commonly used during the postoperative period include deep breathing exercises, early ambulation, positioning, huffing, and coughing $[2,8]$. The author Dias C.M et al stated that SBE could be more effective than deep breathing exercises and incentive spirometry in preventing atelectasis and improving gas exchange during the postoperative period [2]. SBE is a breathing exercise which helps to improve and maintain
Rekha B Marbate*, Abhijit D Diwate and Arijit K Das

Department of PT in Cardiovascular and Respiratory science; DVVPF'S COPT, Ahmednagar, MUHS, Nashik, India

*Author for correspondence:

rekha.matbate@gmail.com 
the volume of breath you take and can be used regularly to clear mucus from airway (secretion clearance) $[9,10]$. It help to Improve depth of breathing, aids secretion clearance, helps improve rib cage mobility and flexibility, stretches respiratory muscles, improves cough strength and effectiveness [11-13].

There have been studies showing SBE can improve peak cough flow or Peak expiratory flow rate (PEFR) in various neuromuscular conditions $[10,14,15]$. Very few studies have been conducted in a cardiac surgery patient. However, there are no studies to prove the efficacy SBE on PEFR in cardiac surgery patient. Hence, we aim to study the immediate effect of SBE on PEFR in cardiac surgery patients.

\section{Methodology}

This pilot study is an experimental study with pre and post-test design. It was carried out in the Cardiac surgery and Rehabilitation unit, Ahmednagar (M.S). Research is duly approved by the Institutional Ethical Committee under MUHS, Nashik (MS) All the patients posted for Cardiac surgery with age group 40-70 years both Males and Females planned for surgery with mediastinal approach were screened pre-operatively for inclusion and patients diagnosed with any neuromuscular condition and having difficulty in cognitive function were excluded. All patients ready to participate was signed informed consent form after the provision of information regarding procedures, benefits and possible complication. Screened subjects developing post-operative complication like:-ventricular arrhythmias, intra-operative myocardial infarction, and severe blood loss $>1500 \mathrm{ml}$. Hemodynamic instability e.g., mean arterial pressure $<70 \mathrm{mmHg}$, reduced output requiring intra-aortic balloon pump or vasoactive drugs. Intubation duration $>72$ hours and need re-intubation were excluded.

\section{Procedure}

Peak Expiratory Flow Rate is a person's maximum speed of expiration, as measured by a small, hand-held device developed by mini-Wright peak flow meter was used to monitor a person's ability to breath out. For healthy Females its values ranging from 380$460 \mathrm{~L} / \mathrm{min}$ and for Males $480-660 \mathrm{~L} / \mathrm{min}$ by EU (European scale) scale. It varies with age and height for both the sexes [13] (FIGURES 1 and 2).

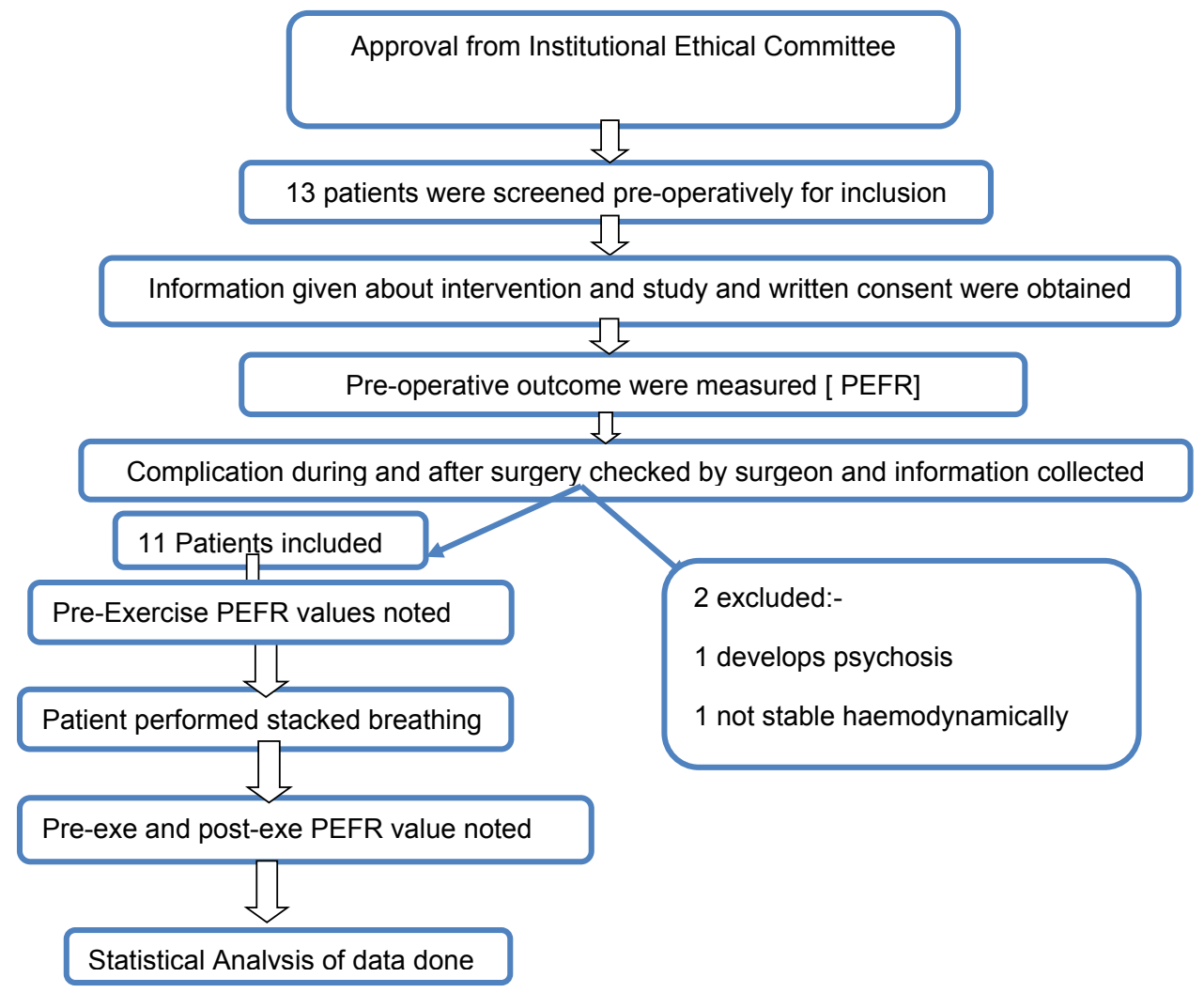


FIGURE 2. Peak expiratory flow rate assessed by peak flow meter.

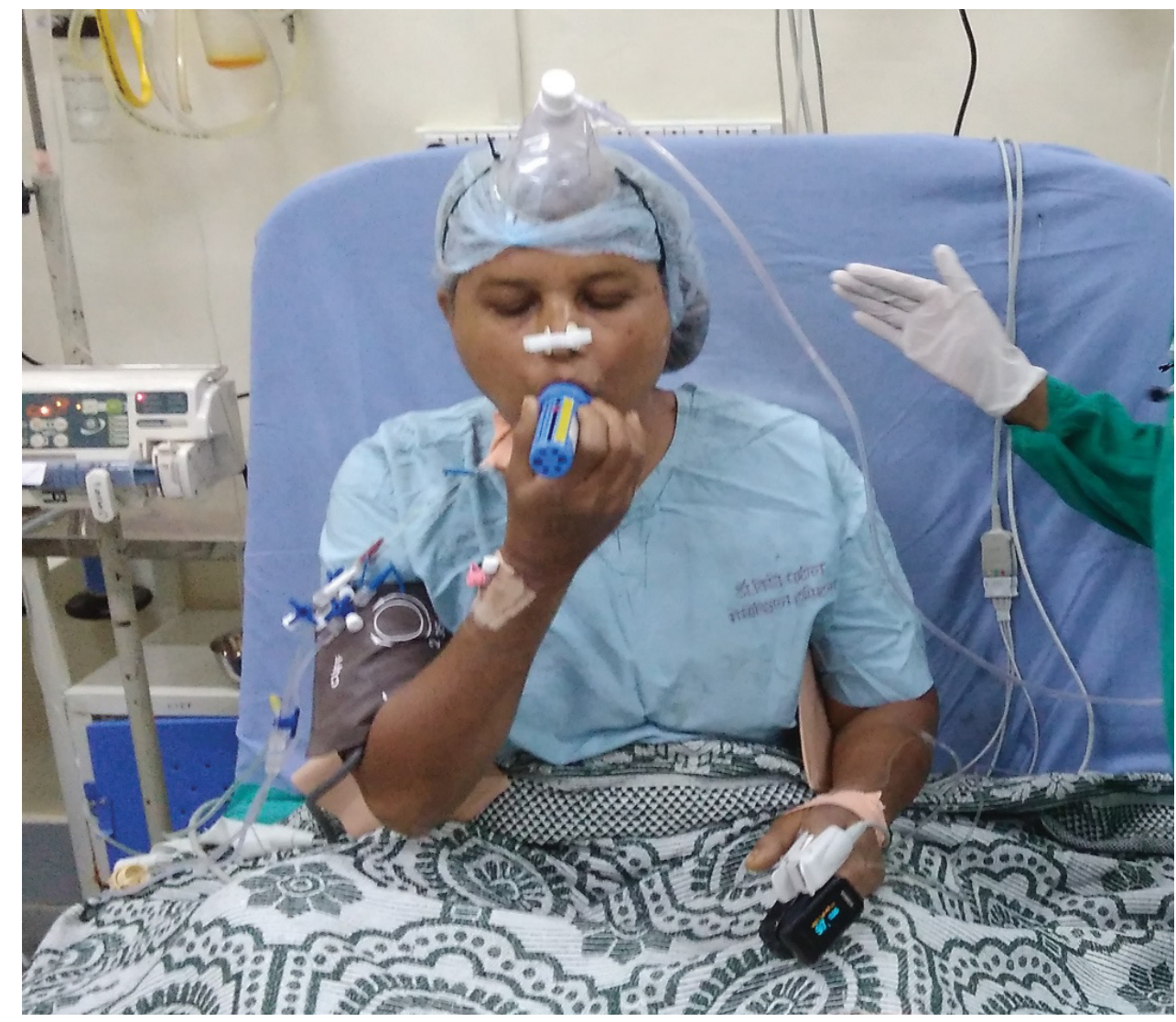

Stacked Breathing Exercise (SBE) was performed previously in different conditions by researchers like e.g. In uchene Muscular Dystrophy patients by Michael $\mathrm{C}$ et al. and physiological acute effects of air stacking exercise on cough peak flow and chest wall volumes of healthy subjects was studied by Sarmento A et al. $[11,14]$. They all mentioned in their study to Hold breath for 20 seconds after maximum inspiratory capacity achieved, which is difficult for the early cardiac surgery patients [11,14]. Hence by considering patient's breath holding capacity on early post-operative days and hemodynamic safety, breath stacking time is revised to 10 seconds for intervention in this study. The patient was positioned in a long sitting position on the bed with continuous monitoring of hemodynamic parameters including SPO2 (oxygen saturation), pulse rate, non-invasive blood pressure, respiratory rate. The patient was instructed to take a deep breath and take 3-4 breath one over another till the fullness of chest experience and was asked to hold the breath for a maximum of 10 seconds then expire by pursed lip breathing or splinted coughing (if secretions are present). There was a gap of 15-30 seconds in each repetition to allow relaxation, 10 repetitions, and twice daily on $1^{\text {st }}, 2^{\text {nd }}$ and $3^{\text {rd }}$ post-operative day. PEFR was checked as per standard guidelines. To restrict the effect of conventional physiotherapy protocol on outcome measures, we will administer the stacked breathing exercise before administrating the conventional therapy protocol (FIGURE 3).

There are a total of six sessions termed as IA, IB, IIA, IIB, IIIA, and IIIB. Preexercise and post-exercise values for PEFR was collected in six treatment session from IA to IIIB ( $\mathrm{A}=$ morning session, $\mathrm{B}=\mathrm{Afternoon}$ session and I, II, III were POD-1, 2 and 3 respectively) for ease of statistical calculations (FIGURES 4 and 5).

\section{Statistical Analysis}

Statistical Analysis done by GraphPad instat software. After passing through Kolmogorov and Smirnov test of normality, repeated measure ANOVA was used for statistical analysis followed by Tukey test.

\section{Result}

Our initial sample size consists of a total of 13 subjects including 7 males and 6 female 


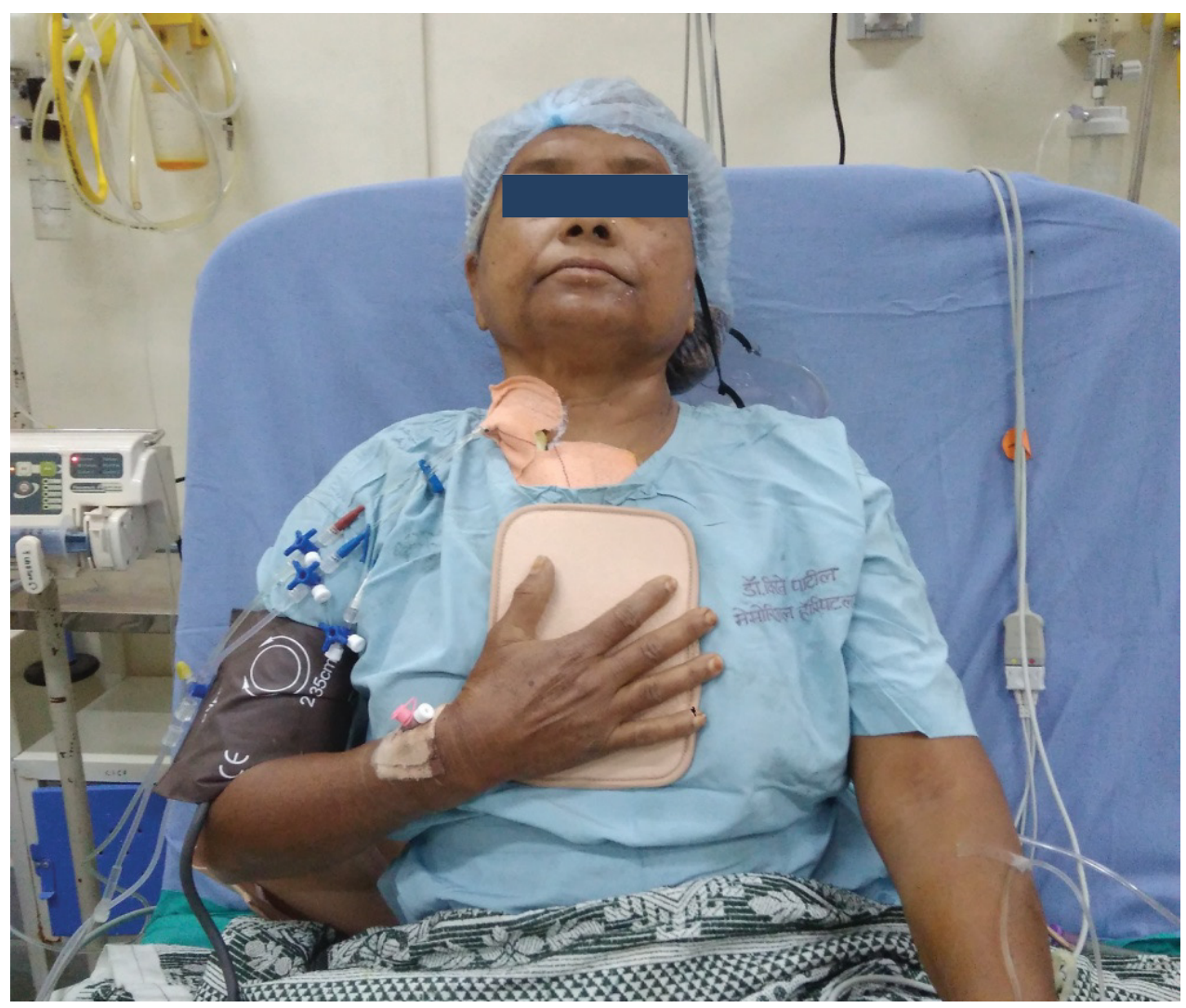

FIGURE 3. Splinted coughing at the end of 10 second hold of maximum breath.

Ask patient to expire by pused lip breathing or splinted coughing if secretions are present

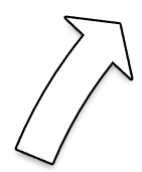

Repeat the procedure for 10 times.

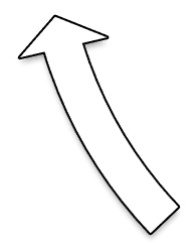

Wait for $15-$ 30 second

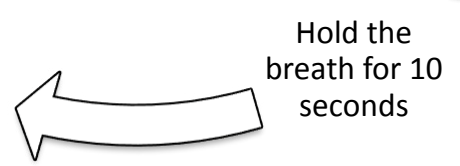

patients for this pilot study evaluated preoperatively. Out of which 1 female patient was excluded (re-intubated) and 1 male not stable hemodynamically. Therefore only 11 patients completed the study. All 11 patients

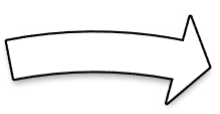

Ask the participant to take a deep breath and hold

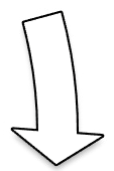

Ask the participant to take 3-4 breath one another and hold for maximum time upto his or her capacity

\section{FIGURE 4. Procedure.}

are extubated on the same day of surgery. There was no statistical difference noted in term of demographic and functional variables in the pre-operative period (TABLES 1 and 2).

FIGURE 3 shows that, improvement in 


\section{Peak expiratory Flow Rate}

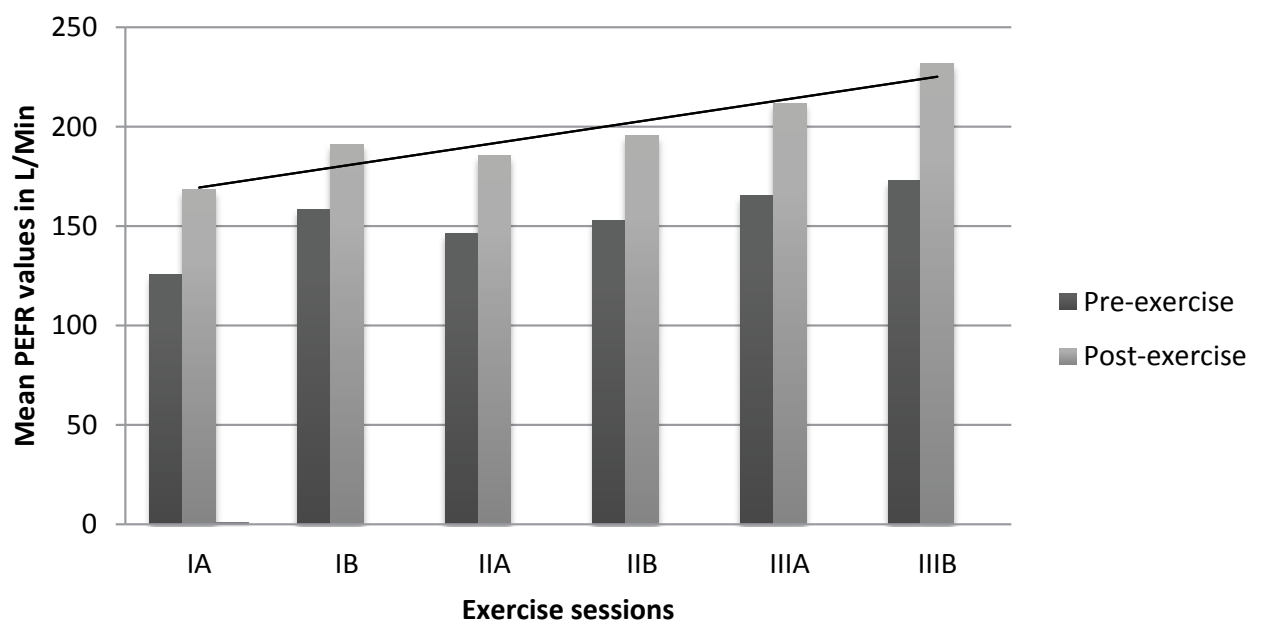

FIGURE 5. Graphical representation of mean PEFR values pre and post SBE session.

\begin{tabular}{|c|c|}
\hline TABLE 1. Demographic and baseline surgical data with baseline outcome measure. \\
Variables & Patient population $\mathbf{n = 1 1}$ Mean \pm SD \\
\hline Age (In years) & $56.18 \pm 12.17$ \\
\hline Male/Female & $6 / 5$ \\
\hline BMI & $22.82 \pm 3.75$ \\
\hline Type of surgery, CABG/ Valve surgery & $9 / 2$ \\
\hline Intubation Period (In Hours) & $8.33 \pm 1.79$ \\
\hline PEFR (In L/Min) & $322.73 \pm 69.15$ \\
\hline (*1 min 30 seconds considers as 1.5 hours) \\
\hline
\end{tabular}

TABLE 2. Comparisons of outcome measurements by Tukey test of multiple variance of analysis.

\begin{tabular}{|c|c|c|c|c|c|}
\hline Comparisons of data & Mean \pm SD & $\begin{array}{c}\text { Mean } \\
\text { difference }\end{array}$ & $\begin{array}{c}\% \\
\text { change }\end{array}$ & Significance & $p$ value \\
\hline \multirow{2}{*}{ IA: pre-exe Vs. post-exe } & pre-exe $=125.45 \pm 40.83$ & \multirow{2}{*}{-42.727} & \multirow{2}{*}{$14.55 \%$} & \multirow{2}{*}{ * } & \multirow{2}{*}{$\mathrm{p}<0.05$} \\
\hline & post exe $=168.18 \pm 52.31$ & & & & \\
\hline \multirow{2}{*}{ IIA: pre-exe Vs. post-exe } & pre-exe $=146.36 \pm 51.23$ & \multirow{2}{*}{-39.091} & \multirow{2}{*}{$11.78 \%$} & \multirow{2}{*}{ ns } & \multirow{2}{*}{$p>0.05$} \\
\hline & post-exe $=185.45 \pm 50.86$ & & & & \\
\hline \multirow{2}{*}{ IIIA: pre-exe Vs. post-exe } & pre-exe $=165.45 \pm 54.28$ & \multirow{2}{*}{-46.364} & \multirow{2}{*}{$12.28 \%$} & \multirow{2}{*}{$* *$} & \multirow{2}{*}{$p<0.01$} \\
\hline & post-exe $=211.82 \pm 54.19$ & & & & \\
\hline \multirow{2}{*}{ IB: pre-exe Vs. post-exe } & pre-exe $=158.18 \pm 47.50$ & \multirow{2}{*}{-32.727} & \multirow{2}{*}{$9.37 \%$} & \multirow{2}{*}{ ns } & \multirow{2}{*}{$p>0.05$} \\
\hline & post-exe $=190.91 \pm 54.85$ & & & & \\
\hline \multirow{2}{*}{ IIB: pre-exe Vs. post-exe } & pre-exe $=152.73 \pm 46.28$ & \multirow{2}{*}{-42.727} & \multirow{2}{*}{$12.27 \%$} & \multirow{2}{*}{ * } & \multirow{2}{*}{$\mathrm{p}<0.05$} \\
\hline & post-exe $=195.45 \pm 54.10$ & & & & \\
\hline \multirow{2}{*}{ IIIB: pre-exe Vs. post-exe } & pre-exe $=172.73 \pm 60.01$ & \multirow{2}{*}{-59.091} & \multirow{2}{*}{$14.60 \%$} & \multirow{2}{*}{$* * *$} & \multirow{2}{*}{$p<0.001$} \\
\hline & post-exe $=231.82 \pm 62.90$ & & & & \\
\hline \multirow{2}{*}{ IA pre-exe Vs. post-exe IIIA } & IA pre-exe $=125.45 \pm 40.83$ & \multirow{2}{*}{-86.364} & \multirow{2}{*}{$25.60 \%$} & \multirow{2}{*}{$* * *$} & \multirow{2}{*}{$p<0.001$} \\
\hline & IIIA post-exe $=211.82 \pm 54.19$ & & & & \\
\hline \multirow{2}{*}{ IB pre-exe Vs. post-exe IIIB } & IB pre-exe $=158.18 \pm 47.50$ & \multirow{2}{*}{-73.636} & $10000 \%$ & $* * *$ & L \\
\hline & IIIB post-exe $=231.82 \pm 62.90$ & & $10.00 \%$ & 列 & $p<0.001$ \\
\hline IA pro pyols noctovollLR & IA pre-exe $=125.45 \pm 40.83$ & 10636 & $2770 \%$ & $* * *$ & \\
\hline IA pre-exe vs. post-exe ilis & IIIB post-exe $=231.82 \pm 62.90$ & -100.30 & $29.17 \%$ & (n) & $\rho<0.001$ \\
\hline
\end{tabular}

Data expressed by mean \pm SD, \% change=absolute value of change in value/average of two value*100, mean difference, $\mathrm{POD}=$ Post-operative day, exe=exercise, pre=before exercise, post=after exercise, ${ }^{*}=$ slightly significant, ${ }^{* *}=$ moderately significant, ${ }^{* * *}=$ very significant

mean PEFR value after each exercise session the post-exercise value shown in IA and IIB from IA to IIIB. There is a statistically with $\mathrm{p}$ value $<0.05$, IIIA with $\mathrm{p}$-value $<0.01$, significant improvement in pre-exercise to IIIB with p-value $<0.001$. While comparing 
IA Pre-exercise to IIIA post-exercise shows significant improvement of $25.60 \%$ with p-value $<0.001$ and IA pre-exercise with IIIB post-exercise shows total improvement of $29.77 \%$ with $p$-value $<0.001$ at the end of sixth (IIIB) treatment session.

\section{Discussion}

The main purpose of this study is to check the immediate effects of stacked breathing exercise on Peak Expiratory Flow Rate (PEFR) following cardiac surgery. All the patients were evaluated under continuous monitoring of hemodynamic parameters and vitals (Heart rate, blood pressure, respiratory rate, oxygen saturation, arterial blood pressure and temperature). Intervention and assessment were carried out with co-operation of cardiac care team (surgeon, anesthetist and nursing team).

A recent study by Antonio Sarmento et al. [20] healthy subjects were analyzed by optoelectronic plethysmography to investigate the immediate effects of air stacking on cough peak flow, operational volume variation. Statistically significant increases in cough peak flow $(\mathrm{p}<0.03)$ and inspiratory capacity $(\mathrm{p}<0.001)$ were found immediately after Air stacking.

Subsequently, a study by Luciana Chiavegato et al, Evaluated the effect of breath stacking on expiratory flow on patients undergoing non-cardiac thoracic surgery. 30 patients were included and post evaluation it shows that the predicted value of Peak Expiratory Flow increased significantly from $46 \% \pm 11$ to $103 \% \pm 11$ after SBE. This improvement in PEFR is due to, air stacking. SBE acts positively on the biomechanical and physiological components with improvement in the peak expiratory flow (PEF) [13,15-17].

In biomechanical component, SBE is effective in improving rib cage mobility and thereby chest expansion in patients [12]. While in physiological component, it promotes deep breathing and maximal inspiration required for forceful expiration during coughing and huffing activity in the process of clearing out of secretions. The same mechanisms of SBE are applicable in cardiac surgery patients, who suffer from chest pain, chest stiffness, restrictive respiratory capacities along with chances of pulmonary complications e.g., atelectasis, the collapse of alveoli and pneumonia, etc, in early post-operative days $[1,5,6,8]$. We studied the immediate effects of SBE and found improvements in PEFR with every session of SBE. A significant improvement of net $29.77 \%$ when analyzed statistically by Tukey test with $\mathrm{p}$-value $<0.001$, in mean PEFR following Stacked Breathing Exercise, when comparing the pre-exercise value of IA (POD-I morning session) with post-exercise IIIB (POD-III afternoon session).

A study done by, Hyun-Gyu Cha [9], proved stacked breathing is an effective treatment tool to improve PCF in an elderly patient with respiratory muscle insufficiency and chest stiffness reducing coughing capacity. PCF is checked by the same procedure as PEFR is measured in our study. So, this study supported our result. Hence, the immediate effect of stacked breathing can improve peak expiratory flow rate by improving tidal volume and chest wall volume to an improved depth of breathing which facilitates the more powerful expiration after a maximum inspiration. Maximal inspiration with 10-second stacking during SBE facilitate the opening of collateral channels, prevents the collapse of alveoli, remove secretions through splinted coughing and improves the functioning of the respiratory system. Hence SBE can be helpful in improving lung function and reducing or preventing respiratory complications. Hence, stacked breathing can help to improve PEFR.

\section{Conclusion}

Based on the statistical analysis, it is concluded that Stacked Breathing Exercise is significantly effective on improving Peak Expiratory Flow Rate, but not effective to improve oxygen saturation at the tissue level. Hence stacked breathing exercise can be an effective tool to improve patients expiratory flow rate and thereby improve the strength of cough and promote easy airway clearance.

\section{Conflict of Interest}

No conflict of interest.

\section{Acknowledgment}

We express our sincere gratitude to those that participated in this study. We are thankful to all cardiac care team members of cardiac surgery and rehabilitation unit, Ahmednagar.

\section{Funding}

Partially funded by Dr. Vitthalrao Vithe Patil Foundation's College of Physiotherapy, Ahmednagar. 


\section{References}

Yendamuri S. General thoracic surgery in India-the time is now. Indian J. Thorac. Cardiovasc. Surg. 34, S2-S3 (2018).

Dias CM, Vieira Rde O, Oliveira JF, et al. Three physiotherapy protocols: Effects on pulmonary volumes after cardiac surgery. J. Bras. Pneumol. 37(1), 54-60 (2011).

Westerdahl E. Optimal technique for deep breathing exercises after cardiac surgery. Minerva Anestesiol. 81(6), 678683 (2015).

Rady MY, Ryan T, Starr NJ. Early onset of acute pulmonary dysfunction after cardiovascular surgery: Risk factors and clinical outcome. Crit. Care Med. 25(11), 1831-1839 (1997).

Tenling A, Hachenberg T, Tydén H, Wegenius G, Hedenstierna G. Atelectasis and gas exchange after cardiac surgery. Anesthesiology. 89(2), 371-378 (1998).

Taggart DP. Respiratory dysfunction after cardiac surgery: effects of avoiding cardiopulmonary bypass and the use of bilateral internal mammary arteries. Eur.
J. Cardiothorac. Surg. 18(1), 31-37 (2000).

WJ Donohue. Postoperative pulmonary complications. When are preventive and therapeutic measures necessary? Postgrad. Med. 91(3), 173-175 (1992).

Brasher PA, McClelland KH, Denehy L, Story I. Does removal of deep breathing exercises from a physiotherapy program including pre-operative education and early mobilization after cardiac surgery alter patient outcomes? Aust. J. Physiother. 49(3), 165-173 (2003).

https://publicdocuments.sth.nhs.uk/ pil3251.pdf

Jeong JH, Yoo WG. Effects of air stacking on pulmonary function and peak cough flow in patients with cervical spinal cord injury. J. Phys. Ther. Sci. 27(6), 19511952 (2015).

Sarmento A, de Andrade AF, Lima ÍN, et al. Air stacking: A detailed look into physiological acute effects on cough peak flow and chest wall volumes of healthy subjects. Respir. Care. 62(4), 432-443 (2017).
Jacquelin M, Andrea A. The expansion of the pulmonary rib cage during air stacking influenced by age in obese women. PLoS One. 9(11), e110959 (2014).

Mridha M, Amin MR. Peak expiratory flow rate (PEFR)-A simple ventilatory lung function test, review article. J. Shaheed Suhrawardy. Med. Coll. 3(2), 4447 (2011).

Michael C, John R, Lavina JI, Michael F. Active lung volume recruitment to preserve vital capacity in Duchenne muscular dystrophy. J. Rehabil. Med. 49, 49-53 (2017).

Torres-Castro R, Vilaró J, Vera-Uribe $\mathrm{R}$, et al. Use of air stacking and abdominal compression for cough assistance in people with complete tetraplegia. Spinal Cord. 52(5), 354-357 (2014).

Massard G, Wihlm JM. Postoperative atelectasis. Chest Surg. Clin. N. Am. 8(3), 503-502 (1998).

Thoren L. Post-operative pulmonary complications: observations on their prevention by means of physiotherapy. Acta Chir. Scand. 107, 193-205 (1954). 